\title{
Information Priority Based Multicast Routing in MANETs
}

\author{
Rajashekhar C. Biradar, Sunilkumar S. Manvi \\ Department of Electronics and Communication Engineering \\ Wireless Information Systems Research Laboratory \\ Reva Institute of Technology and Management, Bnaglore-560064 \\ E-mail: \{raj.biradar, sunil.manvi\}@revainstitution.org
}

\begin{abstract}
The routing efficiency in Mobile Ad Hoc Networks (MANETs) suffers from frequent battery drains, mobility and large variation of received signal strength. Thus, nodes and links in MANETs become more vulnerable and unstable. Multicast routing in MANETs for group communication requires establishment of reliable links between neighbouring nodes called as reliability pair beginning from the source and extending such reliability pairs enroute to the destination. If there are multiple paths from source to every multicast destination, the reliability of source information may be enhanced by sending higher priority information on higher priority path.

In this paper, we propose a scheme of Information Priority based Multicast Routing in MANETs using multiple paths (IPMRM). IPMRM operates in following phases. (1) Reliability pair factor computation based on node power level, received differential signal strength between the nodes and mobility. (2) Pruning neighbor nodes that have reliability pair factor lesser than a threshold. (3) Finding multicast mesh routes with multiple paths to a destination using request and reply packets. (4) Priority assignment to multiple paths based on minimum value of reliability pair factor of a path and information transfer from source to multicast destination. (4) Route maintenance against link failures. The simulation results for packet delivery ratio and control overhead demonstrate better performance of IPMRM over On Demand Multicast Routing Protocol (ODMRP) and Enhanced ODMRP (EODMRP).
\end{abstract}

Keywords: MANET, neighbor node selection, reliability pair factor, multiple multicast routes.

\section{Introduction}

Wireless links in Mobile Ad Hoc Networks (MANETs) are unstable due to large variation of channel characteristics. To enhance routing efficiency, one needs reliable routes which may be realized with the help of multiple paths. The routing scheme must ensure that the nodes which participate in route establishment and route maintenance have links with higher reliability. Thus, reliable routing protocol design is a challenging research problem in MANETs [1][2]. Reliability of links depends on node power, received signal strength, mobility and physical conditions. The group communication services such as audio/video conference and surveillance applications require reliable nodes on the multicast path for continuous transfer of prioritized and non-prioritized packets.

Various multicast routing protocols proposed in the literature are classified according to two different criteria: (1) proactive and reactive and (2) tree-based or mesh-based. Proactive protocols maintain routing state, while reactive protocols reduce the impact of frequent topological changes by redefining routes on demand. Tree-based schemes establish a single

DOI : 10.5121/ijwmn.2011.3314 
International Journal of Wireless \& Mobile Networks (IJWMN) Vol. 3, No. 3, June 2011

path between any two nodes in the multicast group and they are bandwidth efficient. However, as mobility increases, link failures trigger reconfiguration of entire tree. Mesh-based schemes establish a mesh of alternate paths interconnecting source and destinations. They provide more reliable paths and are more resilient to link failures as well as to various mobility conditions of nodes.

We need reliability in MANET applications such as disaster recovery operations, battlefield scenarios, on line education, etc. In such applications, nodes may achieve reliability through the redundancy that exists in sensing or communicating nodes. However, the redundancy is not provided in routing data from node to sink in a sensor network (a type of MANET). The routing algorithms must take care of redundancy while routing data in a multihop mobile network. Thus, the proposed work provides a better multicast routing scheme using prioritized multiple paths as redundant paths.

\section{Related Works}

To provide higher reliability in routing multicast packets, multiple paths with different priorities may be a better choice such that data are routed on one of the priority paths according to significance of the data (i.e., higher significant data may be sent on top priority path). The work given in [3] proposes that the neighbor node stability is dependent upon link lifetime estimation where received signal strength of a node is monitored continuously using Newton Interpolation to estimate link lifetime. In [4], a signal strength based neighbor selection procedure is implemented to test the causes for failures of routing protocol due to fading channels and unreliable network links. In [5], a QoS (Quality of Service) aware routing problem is proposed by maximizing the link stability and lifetime while minimizing the link cost. Agent based reliable ring multicast routing scheme is proposed in [6] that employs reliable ring backbone construction with the help of convex hull algorithm and multicast routes are established using the backbone. The work given in [7] uses a mesh structure, which is capable of quick recovery from mobility by utilizing node locality to reduce the overhead of route failure recovery and mesh maintenance. The signal strength based neighbor node selection mechanisms and the extension of link lifetime are discussed in [3][4][5]. The work given in [8] proposes two topology control algorithms - ABsolute Distance-based (ABD) and PRedictive Distance-based (PRD), which adjust the transmission range of individual nodes in a MANET to achieve good network throughput in a vehicular environment.

New technical trends such as overlay multicast, network coding-based multicast, energy-efficient multicast etc., are discussed in [1]. Reliable multicast transport protocol for MANETs presented in [9] recovers data from various types of losses using Reliable Adaptive Congestion controlled Transport protocol (ReACT) which combines source-based congestionand error control with receiver-initiated localized recovery. The work given in [10] proposes a mesh-based multicast routing scheme that finds stable multicast path from source to receivers. The multicast mesh is constructed by using route request and route reply packets with the help of multicast routing information cache and link stability database maintained at every node. The work given in [11] characterizes the performance of multicast protocols over a wide range of MANET scenarios. The work given in [12] provides the effects of three mobility models in MANETs. An efficient loss recovery scheme for reliable multicast (CoreRM) is proposed in [13]. The cooperative communications idea is used to support the local loss recovery in multicast. A receiver node experiencing a packet loss tries to recover the lost packet through progressively cooperating with neighboring nodes, upstream nodes or even source node. The 
authors in [14] propose a multipath routing framework for the Internet access through Wireless Mesh Networks (WMNs) by using multiple access routers.

In ODMRP [15], a source floods request packets periodically and receiver responds to the packet by using backward learning. The nodes on the path from the receiver to source form a mesh of forwarding nodes. Advantage of ODMRP is that it produces high packet delivery ratio under mobility conditions since it reduces the overhead due to re-establishment of routes under route failures. Disadvantage of ODMRP is the growth of control overhead with network size. Enhanced ODMRP with Motion Adaptive Refresh (EODMRP) given in [16] presents an enhancement of ODMRP with refresh rate dynamically adapted to the environment. An additional enhancement is unified local recovery and receiver joining. On joining or upon detection of a broken route, a node performs an expanding ring search to graft to the forwarding mesh. EODMRP reduces the overhead up to $901 \%$ yet keeping similar packet delivery ratio compared to the original ODMRP. Other mesh-based protocols include Forwarding Group Multicast Protocol (FGMP) [17], Core Assisted Mesh Protocol (CAMP)[18], location-based multicast protocol[19], and Dynamic Core-based Multicast Protocol (DCMP)[20]), Dynamic Counter-Based Forwarding Scheme for ODMRP (CODMRP)[21] and Resilient On Demand Multicast Routing Protocol (RODMRP)[22].

The work given in this paper is an extension of works given in [23][24]. The scope of the work was limited to selection of reliable neighbor nodes for routing in MANETs and it lacked detailed formulation of components of the scheme. Our contributions in this paper are as follows. (1) selection of reliable neighbor nodes based on reliability pair factor, (2) multiple path discovery for multicast routing through non-pruned neighbors, (3) assignment of priority multiple paths from source to every multicast destination to forward prioritized source information, (4) route maintenance scheme to handle link and node failures and (5) validating the performance of the scheme for packet delivery ratio and control overheads.

\section{Identification of Reliable Neighbor Node}

Reliable neighbor node selection mechanism is given in our previous work [23] and the concept is used for establishing multipath multicast routes using reliability pair. Reliability pair is defined as a set of two connected reliable nodes. Reliability Pair Factor $\left(\mathrm{R}_{\mathrm{PF}}\right)$ defines the link connectivity status. In order to compute $\mathrm{R}_{\mathrm{PF}}$, let us assume $\mathrm{BF}$ as the full battery capacity of a node, then remaining battery power of node $i$ at time $t\left(\mathrm{R}_{\mathrm{i}}^{\mathrm{rem}}(\mathrm{t})\right)$ is given by equation 1 .

$$
R_{i}^{r e m}(t)=R_{j}^{r e m}(t-1)-P_{b} \times N B(t-1, t)-P o w_{I}(t-1, t)
$$

where $P_{b}$ is the power required to transmit a bit, $N B(t-1, t)$ is the number of bits transmitted from time $t-1$ to $t$ and $P o w_{I}(t-1, t)$ is the power required to perform node $i$ is internal operations for the duration $t-1$ to $t$. At $t=0, R_{i}^{\text {rem }}(t)=B F$. It is assumed that $R_{i}^{\text {rem }}(t)$ lies in two ranges based on Power ratio defined by equation 2.

$$
\text { Power ratio }=\frac{\mathrm{R}_{\mathrm{i}}^{r e m}(t)}{B F}
$$

such that $R_{i}^{\text {rem }}(t)$ of node $i$ is either in Low range (if the power ratio is within 101\%) or in High range (if the power ratio is within 10\\%). 
The ranges of $R_{i}^{r e m}(t)$ of node $i$ decides transmission power and connectivity status of reliability pair nodes. Reliability pair is augmented as follows. Let initial positions of nodes $i$ and $j$ have coordinate values $(x 1, y 1)$ and $(x 2, y 2)$, respectively located at a distance $d$ meters apart. At time $T=0$, the distance between the nodes is $d_{(i j, 0)}$, and at time $T=t$, nodes move to new positions $\left(x l^{\prime}, y l^{\prime}\right)$ and $\left(x 2^{\prime}, y 2^{\prime}\right)$ with new distance between them as $d_{(i j, t)}$. The successful transmission of packets between nodes $i$ and $j$ is defined with $\mathrm{R}_{\mathrm{PF}}$ which is directly proportional to the minimum remaining battery power level of either nodes $\left(R_{i}^{r e m}, R_{j}^{r e m}\right)$, differential signal strength $\left(D_{S}\right)$ calculated at either node and inversely proportional to the distance between them $\left(d_{(i j, 0)}, d_{(i j, t)}\right)$. The $\mathrm{R}_{\mathrm{PF}}$ at $T=0$ is given by equation 3 .

$$
\mathrm{R}_{\mathrm{PF}}=\mathrm{K} \frac{\operatorname{Min}\left(\mathrm{R}_{\mathrm{i}}^{\text {rem }}, \mathrm{R}_{\mathrm{j}}^{\text {rem }}\right)+D_{S}}{\mathrm{~d}_{(\mathrm{i}, 0)}}
$$

where $K$ is a proportionality constant. At $T=t$, the $R_{P F}$ is obtained by replacing $d_{(i j, 0)}$ by $d_{(i j, t)}$ in equation 3 .

\section{Information Priority Based Multicast Routing}

Information Priority based Multiple path Multicast routing in MANETs (IPMRM) is constructed using neighbor node selection method. The system operates in the following phases. (1) Pruning neighbor nodes and multicast mesh route discovery, (2) priority assignment to multiple paths and information transfer and (3) route maintenance.

\subsection{Neighbor Node Pruning and Route Discovery}

Initially, the node willing to initiate route set up, identifies its neighbors. The $R_{P F}$ corresponding to each neighbor is computed using equation 3 . We wish to establish multicast routes using nonpruned nodes that have $R_{P F} \geq R_{P F}^{T h}$. Here, $R_{P F}^{T h}$ is the threshold of $R_{P F}$ and is fixed by system administrator required for an application and the routes are established through only nonpruned neighbors.

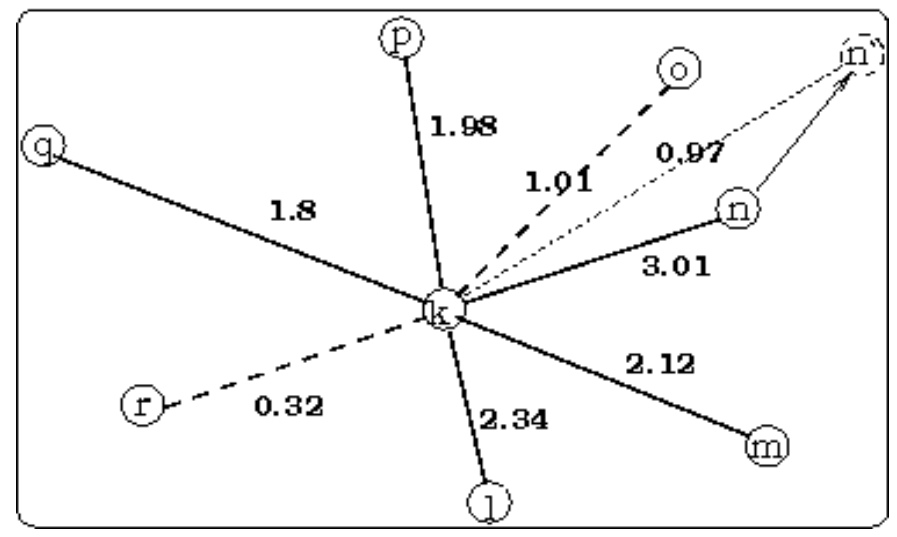

Figure 1: Reliability pair: before and after mobility of node $n$ 
Let us illustrate neighbor selection and pruning by considering node $k$ with seven neighbors $l, m, n, o, p, q$ and $r$ that have 2.34, 2.12, 3.01,1.01, 1.98, 1.8 and 0.32 values for $R_{P F}$, respectively as shown in Figure 1 . At time $T=0$, assume all nodes are stationary with sufficient bandwidth available to exchange hello messages and identify their neighbors. Assume the value of $R_{P F}^{T h}=1.2$ set is set. Node $k$ prunes neighbor nodes $m$ and $q$ since these nodes have $\mathrm{R}_{\mathrm{PF}} \leq R_{P F}^{T h}$. This procedure is repeated at non-pruned neighbor nodes to find their non-pruned neighbors till a destination is reached. For route construction period, assume the node's remaining power $R_{i}^{\text {rem }}$ remains constant (i.e., the nodes are not involved in transmitting/receiving any packets) and there is no node mobility.

At time $T=t$, node $n$ moves to a location $n^{\prime}$ away from node $k$, thus $R_{P F}$ of the node pair $k$ and $n^{\prime}$ decreases to 0.97 because of increase in distance between the nodes for the same remaining power. $R_{P F}$ of node pair $k$ and $n^{\prime}$ becomes less than $R_{P F}^{T h}$, hence the node $n^{\prime}$ in new position will be pruned.

\subsection{Multicast Mesh Route Discovery}

The components of request packet (RQ) and reply packet (RP) that are used to establish multicast routes are shown in Table 1. The components are explained.

\section{RQ Packet Details:}

- Source address $\left(S_{\text {addr }}\right)$ : It is the address of source willing to set up multicast mesh route to group members.

- Multicast address $\left(M C_{a d d r}\right)$ : It is the group address.

- Sequence number (Seq no): It is the $R Q$ packet number originated at source to establish a path and helps in identification of duplicate $R Q$ packets.

- Request flag (RQF): This one bit flag identifies $R Q$ packet. $R Q F=1$ implies $R Q$ packet.

- Path information $\left(P T_{\text {inf }}\right)$ : This field stores node addresses a packet has visited in sequence. It stores all node addresses the packet visits while traveling from source to destination, i.e., visited node appends its address to this field at tail end of the field.

- $\quad R_{P F m i n}$ : This is the minimum value of $R_{P F}$ for a path. The field is updated (at every visited node) if previous node $R_{P F}\left(R_{P F P r e v n o d e}\right)$ is less than $R_{P F m i n}$. It helps in deciding the priority level of a path. This is updated at every visited node.

- Hop count $(H C)$ : It is set to maximum value (number of hops $R Q$ packet can travel) by source. The value is decremented by one for every visited node. This helps in preventing orphan packets, i.e., with hop count as zero, packet is discarded and the source of the packet is informed about it. 
Table 1: RQ/RP Packet Details

\begin{tabular}{|l|l|l|l|}
\hline \multicolumn{2}{|c|}{ RQ Packet } & \multicolumn{2}{c|}{ RP Packet } \\
\hline Parameter & \multicolumn{1}{|c|}{ Value } & Parameter & \multicolumn{1}{c|}{ Value } \\
\hline$S_{\text {addr }}$ & 128.54 .22 .3 & $S_{\text {addr }}$ & 128.54 .22 .3 \\
$M C_{\text {addr }}$ & 228.45 .63 .4 & $M C_{\text {addr }}$ & Same as in RQ \\
& & $D N_{\text {addr }}$ & 124.12 .83 .25 \\
Seq no. & 2 & $S e q$ no. & 4 \\
$R Q F$ & 1 & $R Q F$ & 0 \\
$P T_{\text {infm }}$ & $128.54 .22 .3,--$, & $P T_{\text {infm }}$ & $128.54 .22 .3,--$, \\
& & &,-- 124.12 .83 .25 \\
$R P_{\text {Fmin }}$ & 3.2 & $R P_{\text {Fmin }}$ & 2.1 \\
$H C$ & 20 & $H C$ & 11 \\
& & & \\
\hline
\end{tabular}

\section{RP Packet Details:}

- Source address $\left(S_{\text {addr }}\right)$ : It is the address of the source (as given in $R Q$ packet) to which $R P$ packet is routed from a destination.

- Destination address $\left(D_{\text {addr }}\right)$ : It is the destination address of a member in a multicast group where $R P$ packet is generated and routed to source.

- Multicast address $\left(M C_{a d d r}\right)$ : It is the group address.

- Sequence number(Seq no): Same as in $R Q$. This helps the source to identify $R P$ for a $R Q$ packet.

- Request flag $(R Q F)$ : Setting $R Q F=0$ implies $R P$ packet.

- Path information $\left(P T_{\text {infin }}\right)$ : Same as in $R Q$. The sequence of addresses stored in this field are used by $R P$ packet to route it to the source from destination node. The route traced is through the sequence of addresses starting from tail end address to front address.

- $R_{P F m i n}$ : This is the final value of a path brought by $R Q$ packet at destination. For $R P$ packet, this value is unchanged until delivered to the source.

- Hop count $(H C)$ : This field is set at destination with total number of addresses in $P T_{\text {infm }}$ brought by $R Q$ packet. It is decremented at every visited node while traveling to source.

There are three types of database maintained at a node: (1) neighbor information $(N I)$ to identify pruned and non-pruned neighbors, (2) temporary information $(T I)$ - it is created temporarily at a node to keep track of $R Q$ packets visited at a node for multicast group. The $T I$ from $R Q$ is purged when respective $R P$ packet visits the node and (3) routing information $(R I)$ it helps in transmitting packets through mesh of multicast routes. 
International Journal of Wireless \& Mobile Networks (IJWMN) Vol. 3, No. 3, June 2011

Table 2: Neighbor Information

\begin{tabular}{|l|l|l|}
\hline Neighbor node & $\boldsymbol{R}_{\boldsymbol{P F}}$ & $\boldsymbol{P N F}$ \\
\hline 128.45 .63 .4 & 2.8 & 0 \\
128.36 .23 .42 & 1.1 & 1 \\
129.0 .0 .99 & 3.1 & 0 \\
128.34 .45 .95 & 2.3 & 0 \\
\hline
\end{tabular}

The components of $N I$ are shown in Table 2. It includes: $R_{P F}$ for all neighbor nodes and a pruned node flag $(P N F)$ that indicate whether the node is either pruned or non-pruned. A pruned node cannot participate in routing.

Table 3: Temporary Information

\begin{tabular}{|c|l|c|}
\hline \multicolumn{1}{|c|}{$\boldsymbol{M C}_{\text {group }}$} & Seq no & \multicolumn{1}{c|}{$\boldsymbol{P T}_{\text {infm }}$} \\
\hline $128.45 .63 .4 \& \&$ & 5 & $128.45 .63 .4,--,--,--, 124.12 .83 .25$ \\
$128.36 .23 .42 \& \&$ & 8 & $128.45 .63 .4,--,--,--, 132.53 .21 .102$ \\
& & \\
\hline
\end{tabular}

The components of $T I$ are shown in Table 3. It includes: $M C_{\text {group }}$ - multicast group address, $\$$ Seq no $\$$ - sequence number and $P T_{\text {infm }}$ - path information. Entries in $N I$ are updated at every node for each received $R Q$ packet. This information is purged when $R P$ packet corresponding to $R Q$ packet is received at the node. Components of routing information (RI) at a node are shown in Table 4. It includes: $M C_{\text {group }}$ - multicast group address, $D N_{a d d r}$ - multicast destination address, Next hop - address of next hop for forwarding data packet, $D N$ - destination node, $R_{P F m i n}$ - the minimum $R_{P F}$ value of a path and Normalized priority $\left(\mathrm{P}_{\mathrm{DN}}{ }^{(\mathrm{N}, \mathrm{i})}\right)$ - the normalized priority of $i^{\text {th }}$ next hop at node $N$ for a $D N$.

In request phase, every node identifies its non-pruned neighbors using $R_{P F}$ in $N I$ and broadcasts $R Q$ packet until either it reaches the other end of the network or hop count is zero. While traversing from source, $R Q$ packet is appended with address of visited node in $P T_{\text {infin }}$ at tail end of $R Q$ packet and $R_{P F m i n}$ of path is updated. For every $R Q$ visited at a node, $T I$ components are updated to keep track of duplicate packets and path information. By the time $R Q$ packet reaches destination, the individual route information such as $P T_{\text {infin }}$ and $\$ R_{P F m i n}$ are available with the $R Q$ packet. During request phase, if a node does not find its non-pruned neighbors (due node and link failures), $R Q$ packet is not re-broadcasted.

Table 4: Routing Information

\begin{tabular}{|c|l|l|l|l|}
\hline MC $_{\text {group }}$ & \multicolumn{1}{|c|}{ DN $_{\text {addr }}$} & \multicolumn{1}{|c|}{ Next hop } & $\boldsymbol{R}_{\boldsymbol{P F m i n}}$ & $\begin{array}{c}\text { Normalized } \\
\text { priority }\left(\boldsymbol{P}_{\boldsymbol{D N}}{ }^{(N, i)}\right)\end{array}$ \\
\hline 224.12 .83 .0 & 138.33 .0 .6 & 138.33 .0 .6 & 2.6 & 0.68 \\
& & 138.33 .1 .6 & 3.8 & 1.0 \\
& & 182.0 .56 .7 & 1.9 & 0.5 \\
& \multirow{2}{*}{129.0 .0 .99} & 129.0 .0 .99 & 1.8 & 0.85 \\
& & 128.34 .45 .95 & 2.1 & 1.0 \\
& -- & - & -- & -- \\
\hline
\end{tabular}


In reply phase, $R P$ packet is generated at each multicast destination and it is routed to $S$ through the path defined in $P T_{\text {infm }}$ brought by $R Q$ packet. At every visited node, $R I$ is updated by $R P$ packet for $D N$, Next hop (using path information) and $R_{P F m i n}$ components. For $R P$ packet traveling from destination to source, $R P$ packet updates Next hop by node address $P T_{\text {infin }}[H C]$. Visited node purges its $T I$ for corresponding $R P$ packet (based on sequence number of $R Q$ and $R P$ packets). If a node does not find its next hop due to node or link failures, $R P$ packet is made to wait for some time at the visited node and after timeout, it can be resent. If a node does not find next hop even after timeout, $R P$ packet is dropped and informed to the destination where $R P$ packet is generated. However, nodes are fairly stable in the path and thus $R P$ packet dropping rate may be low since it uses reliable non-pruned nodes.

\subsection{Multiple Path Priority Assignment and Information Transfer}

After receiving $R P$ packet from each multicast destination, source assigns priority to all multiple paths to a destination. Sequence of operations performed by source are as follows: (a) receive $R P$ packets from all destinations, (b) assign normalized priority to multiple paths based on $R_{P F m i n}$ brought by $R P$ packets, (c) segregate information to be transmitted to multicast destinations into different streams and assign the priority level to each stream and (d) transmit high priority information on high priority path and subsequent priority information on next priority paths.

The normalized priority of $i^{\text {th }}$ next hop of a node $N$ for a destination $D N$ is computed as, $\mathrm{P}_{\mathrm{DN}}{ }^{(\mathrm{N}, \mathrm{i})}=\frac{\mathrm{F}_{\mathrm{RPmin}}{ }^{(N, i)}}{\mathrm{F}_{\mathrm{RPmin}}{ }^{(N, H)}}$, where $0<\mathrm{P}_{\mathrm{DN}}{ }^{(\mathrm{N}, \mathrm{i})}<1 . F_{R P \min }{ }^{N, i}$ is $R_{P F m i n}$ of $i^{\text {th }}$ next hop of a node $N$ and $F_{R P m i n}^{N H}$ is highest $R_{P F m i n}$ of all next hop nodes of node $N$.

Figure 2 shows an arbitrary network topology with three multicast destinations $(D 1, D 2$ and $D 3)$ and one source $(S)$ having multiple paths to each destination.

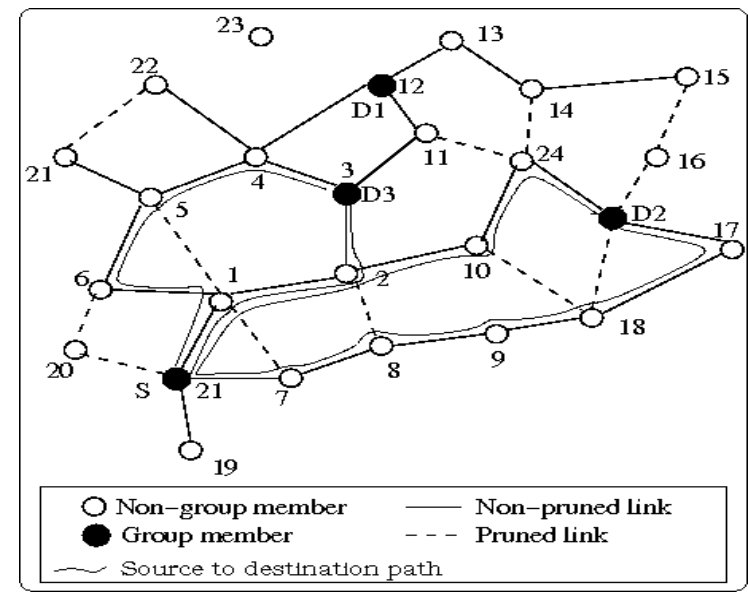

Figure 2: Multiple Priority Multicast Routes

The multicast routing scheme balances various streams on different priority paths (mesh routes) so as to reduce the burden on a single route. As the number of multiple paths to a destination increase, the route reliability increases since different streams flow on different priority paths. 
International Journal of Wireless \& Mobile Networks (IJWMN) Vol. 3, No. 3, June 2011

\subsection{Route Maintenance}

Due to node mobility and node/link failure, a link may be unavailable. This situation can be handled by either of the following mechanisms: (1) local recovery of failed link through nonpruned neighbor nodes and (2) entire reconstruction of routes. Whenever a link fails on a path from source to destination, the failed link divides the path into two segments: one segment is from source to the source side node of a failed link and the other segment is from receiver to the receiver side node of a failed link.

In local recovery mechanism, RQ packet is generated by the source side node of a failed link using source address as its own address and destination address as the receiver side node of a failed link. Route discovery takes place as explained in section 4 to patch-up the path between nodes of a failed link. However, the receiver side node generates only one RP packet needed to patch-up the failed link. If many links fail simultaneously, mesh routes are reconstructed from source to destination since local recovery overhead will be more than overhead of reconstruction of routes.

Whenever a new node wishes to join multicast group, it uses $R Q$ and $R P$ packets to join to multicast group using its non-pruned neighbors existing on multipath. Whenever a node is willing to leave the group, it simply does not respond to any control messages ( $R Q / R P$ packets) and its neighbor nodes remove this node from their list when the network is refreshed.

\section{Simulation Model}

The routing scheme is simulated in various network scenarios to assess the performance and effectiveness of the approach. Simulation environment for the proposed work consists of five models. (1) Network model: An ad hoc network is generated in an area of $l \times b$ square meters. It consists of $N$ number of mobile nodes that are placed randomly in an area. Every node sets $R_{P F}^{T h}$. (2) Power model: Every node is assumed to have its remaining battery power in two randomly distributed ranges, Low range and High range. A node has full battery power, $B F$. $P_{b}$ is the power required to transmit a bit and $P o w_{I}$ is the power required to perform node's internal operations. Differential received signal strength $D_{S}$ is randomly distributed. (3) Propagation model: Free space propagation model is used with propagation constant $\beta$. Transmission range of a node is $r$ for one-hop distance. (4) Mobility model: Random waypoint $(R W P)$ mobility model based upon three parameters is used where speed of movement, direction and time of mobility. In $R W P$, each node picks a random destination within a geographical area, and travels with an average velocity $v$. Node pause time at destination is $Z$. Eight directions are considered for node movement: east, west, north, south, north-east, northwest, south-east and south-west and (5) Traffic model: A multicast group may comprises of $M$ members. Constant bit rate model is used to transmit constant sized packets, $\operatorname{Tr}_{p k t s}$. Coverage area around each node has a bandwidth, $B W_{\text {single-hop }}$, shared among its neighbors.

\subsection{Simulation Procedure}

The proposed scheme is simulated using following simulation inputs. $l=750$ meters, $b=750$ meters, $N=50$ to 200. $R_{P F}^{\text {Th }}=1.2$, Low range $=0$ to $100 \mathrm{~mW}$, High range $=100$ to $1000 \mathrm{~mW}$, $D_{S}=10$ to $100 \mathrm{nW}, B F=1000 \mathrm{~mW}, P_{b}=10 \mathrm{nW}$, Pow $=20 \mathrm{nW} . \beta=2.5, r=350$ meters, $v=10 \mathrm{~m} / \mathrm{s}$, $Z=0.1 \mathrm{~ms}, M=5$ to $25, T_{p k t s}=$ in multiples of $1000, B W_{\text {single-hop }}=20$ Mbps. 
The following performance parameters are assessed.

- Packet Delivery Ratio (PDR): It is defined as the average of ratio of packets received at the destinations to the number of packets sent from a source.

- Control Overhead: It is defined as the number of control packets ( $R Q / R P$ packets) needed to establish routes to all destinations from a source.

- Memory Overhead: It is the total number of bytes to be stored in node database (RI and $N I$ ) to establish and maintain the routes for a multicast group.

- Message Overhead: It is the average number of messages exchanged between every non-pruned node and its neighbors at any given time to maintain neighbor information.

\section{Results}

The simulation is carried out on Pentium IV machine using ' $\mathrm{C}$ ' language. The analysis of performance parameters are given in this section.

Effect of group size on $P D R$ is shown in Figure 3 for various mobility patterns. For static pattern of nodes (no mobility), $P D R$ is fairly high compared to mobility of $5 \mathrm{~m} / \mathrm{s}$ and 10 $\mathrm{m} / \mathrm{s}$. The fall in $P D R$ is due to change of non-pruned node's pattern under mobility condition, thus creating different set of end-to-end multipaths. In all cases, $P D R$ increases with increase in group size as multiple paths to every destination increase and these multiple paths carry more number of priority streams that helps to increase $P D R$.

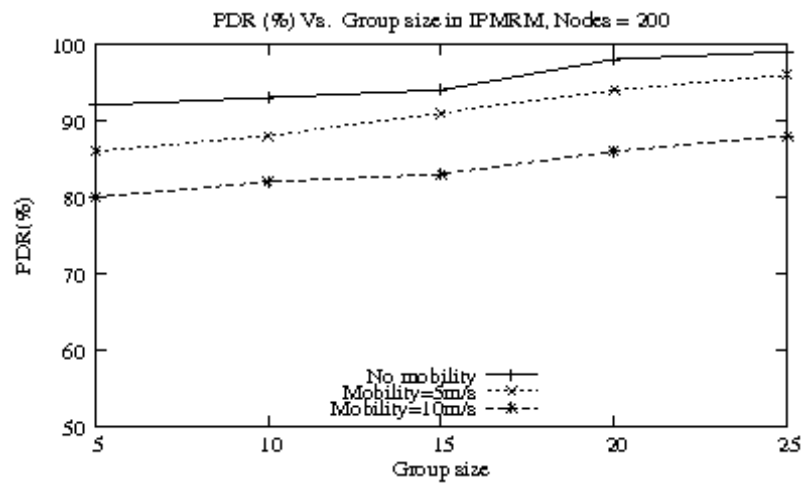

Figure 3: PDR Vs. Group size

$P D R$ for IPMRM is better compared to ODMRP and EODMRP since these protocols do not consider multiple paths to send prioritized data streams and multiple paths are formed based on non-pruned nodes concept (see Figure 4). ODMRP and EODMRP rely on links which are vulnerable to packet drops in contrast to IPMRM that are established based on high reliability. The gap between $P D R$ curve of IPMRM and ODMRP decreases with increase in group size since connectivity improves in $O D M R P$ with increase in group size. But IPMRM maintains consistent $P D R$ with increase in group size. 
International Journal of Wireless \& Mobile Networks (IJWMN) Vol. 3, No. 3, June 2011

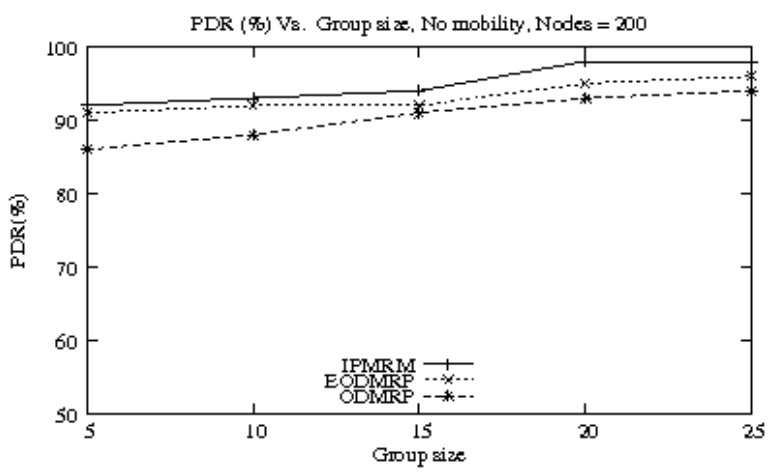

Figure 4: PDR Vs. Group size (comparison with ODMRP and EODMRP)

Figure 5 shows control overhead with increase in number of nodes. For given number of nodes, the control overhead (control packets) increase with increase in group size. As network size increases (beyond 150 nodes), the control overhead decreases at higher multicast group sizes. This is due to the possibility of establishing multiple paths by many number of same non-pruned nodes to destinations since these non-pruned nodes happen to be on the path for all those destinations.

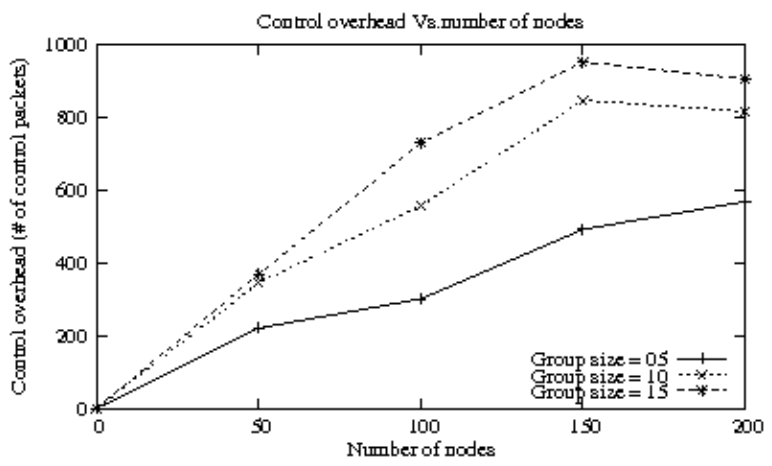

Figure 5: Control overhead Vs. Number of nodes

Route establishment in IPMRM uses only non-pruned nodes and it needs less number of control packets ( $R Q / R P$ packets) compared to ODMRP and EODMRP as these protocols use all the nodes for route establishment (see Figure 6). As number of nodes increase, the overhead increases but the increase is less steeper in IPMRM.

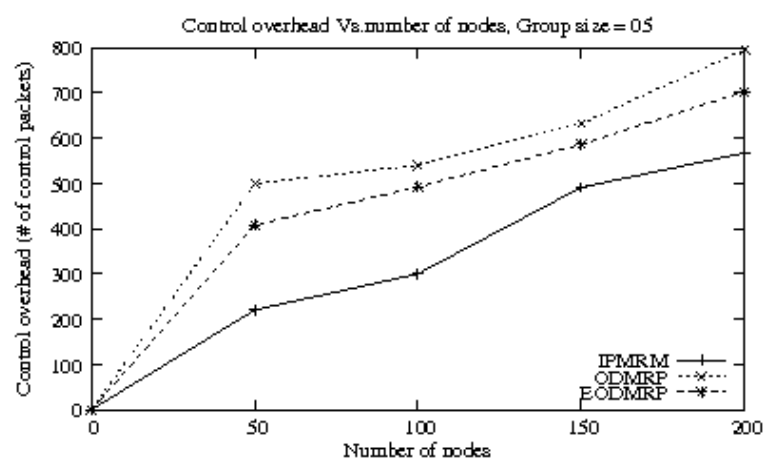

Figure 6: Control overhead Vs. Number of nodes (comparison with ODMRP and EODMRP) 


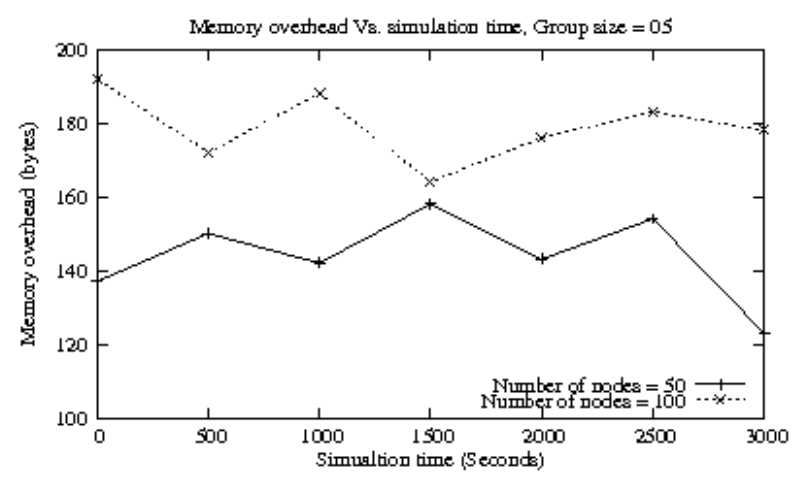

Figure 7: Memory overhead Vs. Simulation time

Figure 7 shows memory overhead of IPMRM for 50 and 100 node topology at discrete simulation time. The overhead in 100 node topology is more than that of 50 node topology. As the number of nodes increase, there are chances that more number of neighbors satisfy $R_{P F}{ }^{T h}$ condition and there are more entries in node database that increases overhead. ODMRP memory overhead is higher than IPMRM (See Figure 8) due to following reasons. (1) Forwarding nodes of $O D M R P$ store join table and join reply packets corresponding to all the neighbors of nodes as there is no node-pruning mechanism on storage as in IPMRM. (2) Number of bytes in join request and join reply packets are more compared to the number of bytes in node database of IPMRM.

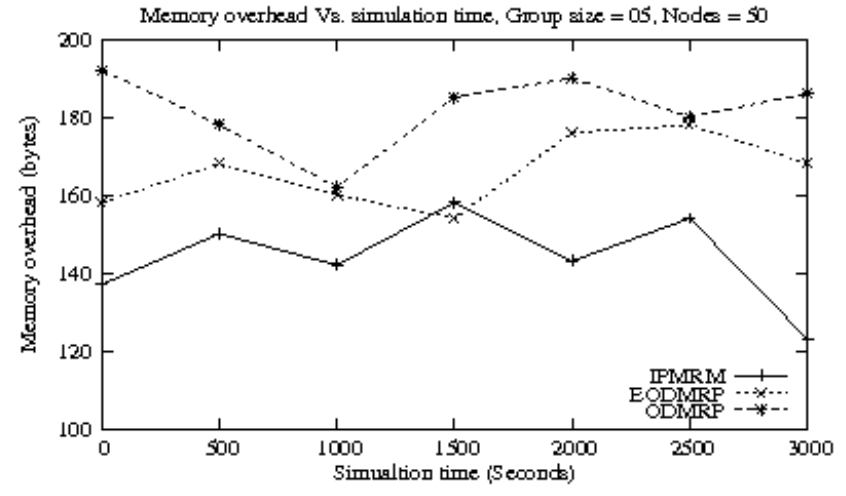

Figure 8: Memory overhead Vs. Simulation time (comparison with ODMRP and EODMRP)\}

However, memory overhead in EODMRP outperforms ODMRP since EODMRP uses expanding ring search mechanism upon joining or detection of a broken route. Expanding ring search mechanism helps EODMRP node to graft to the forwarding mesh there by reducing memory overhead under above said conditions.

Messages exchanged among neighbors to find non-pruned nodes in IPMRM are shown in Figure 9 for 50 and 100 node topology over a simulation period. Message overhead in 100 node topology is more than that of 50 node topology as more messages are needed to select non-pruned nodes. 


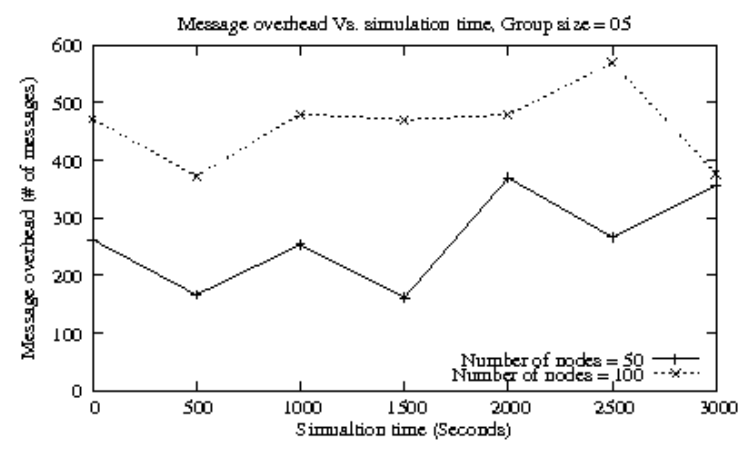

Figure 9: Message overhead Vs. Simulation time

Because of fixed duration refreshing in $O D M R P$, messages exchanged to set up a mesh of routes is high for short refresh duration due to frequent mobility and route breaks whereas in $I P M R M$, the route breaks are less frequent since it uses multiple routes to send prioritized data; if any route breaks, then other less priority path is used to send the data (see Figure 10). Since EODMRP uses dynamic refreshing and local recovery of routes, messages exchanged are less compared to ODMRP.

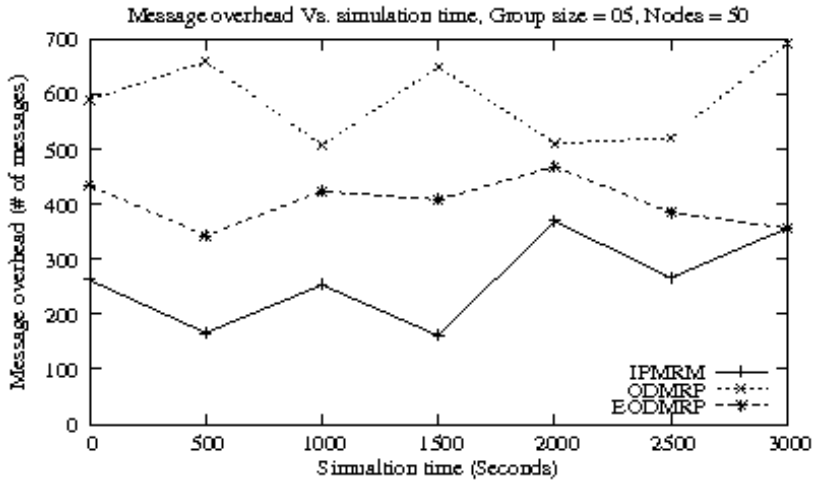

Figure 10: Message overhead Vs. Simulation time (comparison with ODMRP and EODMRP)

\section{Conclusions}

In this paper, we proposed a scheme for information priority based multiple path multicast routing in MANETs that used reliable neighbor node selection mechanism. Neighbor nodes are selected that satisfy certain threshold of reliability pair factor to find non-pruned neighbors. Non-pruned neighbors are used to establish reliable multipath multicast routes with assigned priority levels using request and reply control packets along with node database comprising of neighbor and routing information. Prioritized multipaths carry various priority data to multicast destinations. Neighbor node selection is realized with the help of node power model and mobility model. Robust route maintenance mechanism is provided to handle link and node failure situations. The results of simulation for packet delivery ratio, different overheads and packet delays illustrate the effectiveness of the developed scheme over well established mesh based multicast routing protocols such as $O D M R P$ and EODMRP. 
International Journal of Wireless \& Mobile Networks (IJWMN) Vol. 3, No. 3, June 2011

\section{References}

[1] Luo Junhai, Ye Danxia, Xue Liu, Fan Mingyu, “A Survey of Multicast Routing Protocols for Mobile Ad-hoc etworks", IEEE Communications Surveys and Tutorials, Vol. 11, No. 1, 2009, pp. 78-91.

[2] Javad Akbari Torkestania, Mohammad Reza Meybodib, "Mobility-Based Multicast Routing Algorithm for Wireless Mobile Ad-Hoc Networks: A Learning Automata Approach", Computer Communications, Vol. 33, No. 6, 2010, pp. 721-735.

[3] Da-peng WU, Mu-qing WU, Yan Zhen, “ Reliable Routing Mechanism Based on Neighbor Stability for MANET", The Journal of China Universities of Posts and Telecommunications, Vol. 16, No. 3, 2009, pp. 33-39.

[4] Kwan-Wu Chin, John Judge, Aidan Williams, Roger Kermode, “Implementation Experience with MANET Routing Protocols", ACM SIGCOMM Computer Communications Review, Vol. 32, No. 5, 2002, pp. 49-59.

[5] Md. Mamun-Or-Rashid, Choong Seon Hong, "LSLP: Link Stability and Lifetime Prediction Based QoA Aware Routing for MANET", Proc. Joint Conference on Communications and Information (JCCI) (2007).

[6] Rajashekhar C. Biradar, Sunilkumar S. Manvi, “Reliable Ring Based Multicast Routing Scheme in MANET: An Agent Based Approach", Proc. IEEE Conference on Automation Science and Engineering (IEEE CASE), Bangalore, India, pp. 507-512, (2009).

[7] Seungjoon Lee, Chongkwon Kim, "Neighbor Supporting Ad Hoc Multicast Routing Protocol", Proc. 1st ACM international symposium on Mobile ad hoc networking and computing, pp. 37-44, (2000).

[8] P. Siripongwutikorn, B. Thipakorn, "Mobility-Aware Topology Control in Mobile Ad hoc Networks", Computer Communications, Vol. 31, No. 14, 2008, pp. 3521-3532.

[9] Venkatesh Rajendran et al. "Combining Source And Localized Recovery to Achieve Reliable Multicast in Multi-Hop Ad-Hoc Networks", Proc. Networking, 2004, pp. 1-12.

[10] Rajashekhar C. Biradar, Sunilkumar S. Manvi, “Link Stability Based Multicast Routing Scheme in MANET", Elsevier Computer Networks, Vol. 54, No. 7, 2010, pp. 1183-1196.

[11] Kumar Viswanath, Katia Obraczka, Gene Tsudik, "Exploring Mesh and Tree-Based Multicast Routing Protocols for MANETs", IEEE Transactions on Mobile Computing, Vol. 5, No. 1, 2006, pp. 28 42.

[12] R. Manoharan, E. Ilavarasan, "Impact of Mobility on the Performance of Multicast Routing Protocols in MANETs", International Journal of Wireless and Mobile Networks, Vol. 2, No. 2, 2010, pp. 110-119.

[13] Mengjie Huang, Gang Feng, Yide Zhang, "Cooperative Loss Recovery for Reliable Multicast in Ad Hoc Networks", International Journal of Communications, Network and System Sciences (IJCNS), No. 3, 2010, pp. 72-78.

[14] Vinh Dien Hoangi, Maode Ma, Hiroshi Harada, "Multi-paths Routing with Load Balancing for Internet Access in Wireless Mesh Networks", International Journal of Wireless and Mobile Networks, Vol. 2, No. 1, 2010, pp. 65-75.

[15] William Su Sung-Ju Lee, Mario Gerla, “On-demand Multicast Routing Protocol in Multihop 
Wireless Mobile Networks", Mobile Networks and Applications, Vol. 7, 2009, pp. 441-453.

[16] Soon Y. Oh, Joon-Sang Park, Mario Gerla, “E-ODMRP: Enhanced ODMRP with Motion Adaptive Refresh", Journal of Parallel and Distributed System, Vol. 64, No. 8, 2008, pp. 1044-1053.

[17] Ching-Chuan Chiang, Mario Gerla, Lixia Zhang, “Forwarding Group Multicast Protocol (FGMP) for Multihop, Mobile Wireless Networks", Cluster Computing, Vol. 1, No. 2, 1998, pp. 187-196.

[18] Ewerton L. Madruga, J. J. Garcia Luna Aceves, ' Scalable Multicasting: The Core-Assisted Mesh Protocol", Mobile Networks and Applications, Vol. 6, No. 2, 2001, pp. 151-165.

[19] Young-Bae Ko, Nitin H. Vaidya, "Geocasting in mobile Ad Hoc Networks: Location-Based Multicast Algorithms", Proc. Second IEEE Workshop on Mobile Computer Systems and Applications, 1999, pp. 101-107.

[20] B. S. Manoj, Subir Kumar Das, C. Siva Ram Murthy, “A Dynamic Core Based Multicast Routing Protocol for Ad Hoc Wireless Networks", Proc. 3rd ACM International Symposium on Mobile Ad Hoc Networking and Computing, 2002, pp. 24-35.

[21] Nian Mei, Wang Neng, “A Dynamic Counter-Based Forwarding Scheme for ODMRP", Proc. International Conference on Wireless Communications, Networking and Mobile Computing (WiCOM), 2006, pp.1-4.

[22] Dhammika Pathirana, “RODMRP - Resilient On Demand Multicast Routing Protocol", A thesis, Rochester Institute of Technology, Feb. 2007.

Available Online: https://ritdml.rit.edu/dspace/bitstream/1850/5288/1/DPathiranaThesis02-2007.pdf

[23] Rajashekhar C. Biradar, Sunilkumar S. Manvi, “'Channel Condition and Mobility Based Choice of Neighbor Node for Routing in MANET", Proc. International Conference on Advances in Computer Engineering, (ACE) 2010, Bangalore, India, pp.74-78, June 21-22, 2010.

[24] Rajashekhar C. Biradar, Sunilkumar S. Manvi, “Reliable Neighbor Based Multipath Multicast Routing in MANETs", Proc. First International Conference on Computer Science and Information Technology (COSIT) 2011, LNCS, pp. 33-43, June 21-22, 2010.

\section{Author Biography of Rajashekhar C. Biradar}

Rajashekhar C. Biradar completed his B.E (Electronics and Communication Engineering) and M.E.(Digital Electronics) from Karnataka University Dharwad, India. He is working as Professor in the Department of ECE, Reva Institute of Technology and Management, Bangalore, India. He has submitted his $\mathrm{PhD}$ thesis to Visvesvaraya Technological University (VTU), Belgaum, India. To his credit, he has several national/international journals and conference publications. His research areas include multicast routing in mobile ad hoc networks, wireless Internet, group communication in MANETs, agent technology. He is a member IETE (MIETE), member IE (MIE) and member ISTE (MISTE), India, member of IEEE (USA), member of ACM (USA).

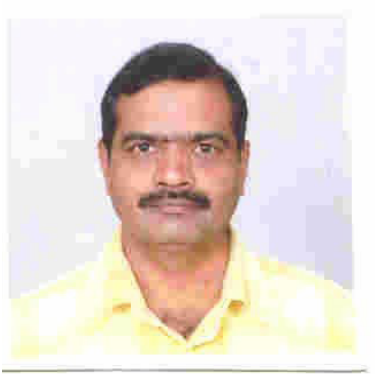




\section{Author Biography of Dr. Sunilkumar S. Manvi}

Sunilkumar S. Manvi received M.E. degree in Electronics from the University of Visweshwariah College of Engineering, Bangalore, $\mathrm{Ph} . \mathrm{D}$ degree in Electrical Communication Engineering, Indian Institute of Science, Bangalore, India. He is currently working as a Professor and Head of Department of Electronics and Communication Engineering, Reva Institute of Technology and Management, Bangalore, India. He is involved in research of Agent based applications in Multimedia Communications, Grid computing, Ad-hoc networks, E-commerce and Mobile computing. He has published about 100 papers at national and

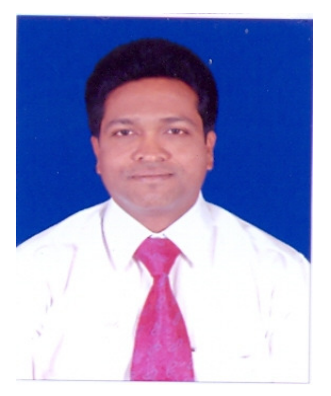
international conferences and 40 papers for national and international journals. He has published 3 books. He is a Fellow IETE (FIETE, India), Fellow IE (FIE, India) and member ISTE (MISTE, India ), member of IEEE (MIEEE, USA), He has been listed in Marqui's Who's Who in the World. 